and manual ventilation of the patient continued throughout transfer. Intravenous phenytoin controlled seizure activity. During these procedures several staples in the scalp lacerations became dislodged and there was profuse bleeding from the wounds. Attempts were made to control the bleeding but haemostasis was not achieved.

On arrival at the neurosurgical unit the patient's condition had deteriorated. She was hypotensive (arterial pressure $70 / 40 \mathrm{~mm} \mathrm{Hg}$ ), tachycardic (heart rate 130 beats/min), and tachypnoeic. Rapid resuscitation was carried out and blood cross-matched. Haemoglobin level was $7 \cdot 1 \mathrm{~g} / \mathrm{dl}$. Over the next two hours she had a further seizure but her haemodynamic status stabilised. She was transfused with four units of packed cells. Subsequent surgery confirmed that the knife had penetrated the dura and there was a small subdural haematoma, which was evacuated. Postoperative care was uneventful and she was discharged on a maintenance dose of phenytoin.

\section{Discussion}

The problems associated with the inter hospital transfer of the head injured patient are well recognised ${ }^{1}$ and haemorraghic shock following scalp lacerations is described. ${ }^{2}$ It would seem obvious to any clinician with experience in emergency departments that scalp lacerations have the potential for profuse blood loss and that their correct management demands secure haemostatic control. This usually involves simple suturing with an appropriate material. Other acceptable methods include surgical staples ${ }^{3}$ or more specialised scalp clips, ${ }^{4}$ the crucial point being that good, secure haemostasis is achieved.

If haemostasis is not achieved a cascade of events can follow, with resultant deterioration in the patient's clinical state. This is especially dangerous during interhospital transfer, which is a common stage in the management of the head injured patient requiring neurosurgical assessment. The head injured patient needs prompt investigation and treatment if secondary insults to the brain are to be avoided. Hence, reducing the period between admission and definitive care should be facilitated by doing the simple things efficiently and to a high standard.

With good technique the time taken to correctly manage scalp lacerations should not in itself introduce a significant delay. It is better to delay transfer and achieve haemostasis, since the clinical deterioration of the patient during transfer could have serious consequences and itself result in significant delay.

We suggest that the potential for scalp lacerations to cause haemorraghic hypotension should be emphasised to all clinicians. Furthermore, if interhospital transfer is indicated the referring clinician must be satisfied that haemostasis is secure. Haemostatic procedures must also be able to withstand the transfer and the management of any complications which could be anticipated. Failure to follow these basic principles may place the patient at increased risk as a result of the need for additional resuscitation, and therefore delay the definitive management.

1 Gentleman D, Dearden M, Midgley S, MacLean D Guidelines for resuscitation and transfer of patients with Guidelines for resuscitation and transfer of
serious head injury. $B M \mathcal{F} 1993 ; 307: 547-52$.

2 Lemos MJ, Clark DE. Scalp lacerations resulting in haemorrhagic shock: case reports and recommended haemorrhagic shock: case reports and

3 Ritchie AJ, Rocke LG. Staples versus sutures in the closure of scalp wounds: a prospective double blind, randomised trial. Injury 1989;20:217-8.

4 Sykes LN, Cowgill F. Management of haemorrhage from severe scalp lacerations with Raney clips. Ann Emerg Med 1989;18:995-6.

Accident and
Emergency
Department,
Stockport Infirmary,
Stockport
M Saab
A Gray
D Hodgkinson
Neurosurgical
Department,
Manchester Royal
Infirmary, Manchester
M Irfan
Correspondence to:
M Saab,
Senior Registrar in Accident
and Emergency, Stockport
Infirmary, Wellington Road
South, Stockport SK1 3UJ,
United Kingdom.

\title{
Warfarin and the apparent minor head injury
}

\author{
M Saab, A Gray, D Hodgkinson, M Irfan
}

Key terms: anticoagulation; apparent minor head injury; intracranial haematoma; warfarin

It is estimated that 1.4 million patients each year attend accident and emergency (A\&E) departments in the United Kingdom with a head injury. The vast majority have a minor head injury which has been defined as a history of unconsciousness of less than 20 minutes, a Glasgow coma scale score of 13-15 on admission, and an inpatient stay not exceeding 48 hours. $^{1}$ Most of these patients are 
discharged home after assessment in the $A \& E$ department but a small proportion will be admitted for observation. Because the indications for the use of anticoagulants are expanding $^{2}$ there is a small but possibly increasing number of patients who present with an apparently minor head injury who are on anticoagulant drugs. These patients are at increased risk of developing intracranial haematoma. ${ }^{2}$ Their management should be different from that of patients with the same type of injury and clinical findings who are not on anticoagulants.

In this paper we present two such cases and suggests an appropriate management plan.

\section{Case reports}

PATIENT 1

A 70 year old woman on long term anticoagulation with warfarin for cardiomyopathy and atrial fibrillation attended the $A \& E$ department complaining of increasing headache. She had fallen from a standing position and hit the back of her head on a pavement two days before presentation. There was no history of loss of consciousness at the time of injury. She had not consulted a medical practitioner because she considered it to be a trivial injury. Over the following two days she had developed increasingly severe headache.

On examination she had a Glasgow coma scale score (GCSS) of 15 and a normal neurological examination. There was a tender occipital haematoma. Her international normalised ratio (INR) was $2 \cdot 5$. In view of her increasingly severe headache a CT scan was done. This showed a large left sided subdural haematoma. She was transferred to the regional neurosurgical unit, where her anticoagulation was reversed. In view of the size of the haematoma, surgical evacuation was performed. Her GCSS remained at 15 before surgery.

She made an uneventful recovery.

\section{PATIENT 2}

A 67 year old man on long term anticoagulation with warfarin for mitral valve disease and atrial fibrillation attended the $A \& E$ department, having fallen at home hitting the back of his head on a tiled kitchen floor. There was no reported loss of consciousness but a short period of post-traumatic amnesia (five minutes).

On presentation he complained of mild occipital headache. He had a GCSS of 15 , normal neurological examination, and a small scalp laceration. This was sutured and haemostasis achieved. Skull $x$ rays showed no fracture. His INR was $4 \cdot 2$. He was admitted for observation simply because he was on anticoagulants. His INR was not reversed.

Two hours after admission he collapsed, with a GCSS of 5 . Computerised tomography (CT) showed a large intracranial haematoma. Neurosurgical intervention was considered inappropriate. He died six hours later.

\section{Discussion}

The risk of intracranial haematoma is increased 10-fold in patients on anti- coagulants. ${ }^{3}$ It is three times more likely to occur in women. ${ }^{2}$ No explanation is given for this, but anticoagulated women outnumber anticoagulated men by three to one. In the majority of patients the intracranial haematoma is spontaneous. In a minority there is a history of preceding head trauma. ${ }^{4}$ This trauma can apparently be minor.

Patients over the age of $50^{5}$ with a duration of treatment of more than one year ${ }^{6}$ are particularly at risk. The higher the value of the INR the greater the risk.

The following plan is suggested for patients with apparent minor head injury who are on anticoagulants:

(1) All these patients should be admitted for standard neurological observation following an apparent minor head injury.

(2) All patients should have their INR checked and the indication for anticoagulation reviewed with the medical team. It has been shown that up to one third of patients do not have a good indication for anticoagulation treatment. ${ }^{2}$

(3) The INR should be adjusted according to the balance between the risk of developing intracranial haematoma and the risk of thromboembolic disease. The risk for intracranial haematoma increases if the INR is greater than 2 . The risk of thromboembolic disease is greater in recurrent pulmonary embolism, the presence of mechanical prosthetic heart valves, and atrial fibrillation with mitral stenosis.

(4) Overanticoagulation (INR outside the desired therapeutic range for the condition) should be corrected with fresh frozen plasma or vitamin $\mathrm{K}$. The advice of a haematologist is recommended.

(5) At present, patients who develop persisting or increasing neurological symptoms or signs after an apparently minor head injury will have an urgent CT scan. ${ }^{7}$ We would recommend that there should be a much lower threshold for requesting a CT scan in patients who are on long term anticoagulants and have sustained an apparently minor head injury. In a developing intracranial haematoma, headache is the most common symptom. We would recommend a CT scan for patients on anticoagulants with persistent headaches unrelieved by simple analgesia.

(6) If patients develop an intracranial haematoma, confirmed on CT scan, then early consultation with a neurosurgical unit and medical department and full reversal of anticoagulants should be arranged promptly.

1 Field JH. The epidemiology of head injuries in England and Wales. London: HMSO, 1976.

2 Mattie H, Kohler S, Huber P, Ronner M, Steinslape KF. Anticoagulation related intracranial extracerebral Anticoagulation
haemorrhage.
$1989 ; 52: 829-37$.

3 Hylek EM, Singer DE. Risk factors for intracranial haematoma in out-patients taking warfarin. Ann Intern haematoma in out-patie

4 Wintzen AR, Tijssen JGP. Subdural haematoma and oral anticoagulant therapy. Arch Neurol 1982;39:69-72.

5 Wintzen AR, de Jonge H, Loeliger EA, Bots GTAM. The risk of intracerebral haemorrhage during oral anticoagulant treatment: a population study. Ann Neurol 1984;16:553-8.

6 Franke CL, de Jonge J, Von Swieten JC, de Coul AAW, Von Gijn J. Intracranial haematomas during anticoagulant treatment. Stroke 1990;21:726-30. 